\title{
Not just correlative: a new pathway defines how an ALDH2 SNP contributes to atherosclerosis
}

\author{
Andrew A. Gibb and John W. Elrod \\ Center for Translational Medicine, Lewis Katz School of Medicine, Temple University, Philadelphia, Pennsylvania, USA.
}

\begin{abstract}
Individuals with the rs671 SNP in the gene encoding aldehyde dehydrogenase 2 ( $A L D H 2)$ are at increased risk of cardiovascular disease (CVD); however, it has been unclear if this mutation contributes to CVD development. In this issue of the $J C I$, Zhong et al. perform an elegant set of experiments that reveal a pathway wherein the ALDH2 rs671 mutant is phosphorylated by AMPK and translocates to the nucleus where it represses the transcription of a lysosomal $\mathrm{H}^{+}$pump subunit that is critical for lipid degradation and foam cell formation, as occurs in atherosclerosis. The discovery of this pathway may explain how subjects harboring ALDH2 rs671 are at a greater risk for numerous other disease states and thereby provide new targets for therapeutic intervention.
\end{abstract}

\section{Genetic risk factors for atherosclerosis}

Atherosclerosis is the leading cause of cardiovascular disease (CVD) and the primary determinant of heart disease and stroke, the top two killers worldwide (1). The high mortality rate associated with atherosclerosis is of particular importance for individuals suffering from familial hypercholesterolemia, a genetic disorder whereby the loss of at least one functional $\mathrm{LDL}$ receptor $(L D L R)$ allele results in the inefficient clearance of circulating cholesterol and aggressive atherosclerotic plaque formation resulting in premature $\operatorname{CVD}(2,3)$. Another genetic determinant that predisposes individuals to CVD is a SNP in the gene encoding the alcohol-metabolizing enzyme aldehyde dehydrogenase $2(A L D H 2)$. Approximately $40 \%$ of East Asians carry this SNP (ALDH2 rs671), which is associated with the phenomenon known as "Asian flush" that occurs with alcohol consumption. While $A L D H 2$ rs671 is associated with an increased risk of atherosclerosis and coronary artery disease (4), it is unknown whether this SNP causally contributes to CVD development.

\section{ALDH2 and atherosclerosis development}

In this issue, Zhong et al. provide important mechanistic evidence to address the role of $A L D H 2$ rs671 in CVD (5). The authors employed mouse genetics and deleted Aldh2 in the Ldlr knockout background ( $\left(\mathrm{dlr}^{-/}\right)$, a commonly employed experimental model of atherosclerosis, and demonstrated that loss of ALDH2 reduced plaque burden and foam cell formation (fat laden macrophages that contribute to plaque generation and growth). This unexpected observation suggested a critical role for ALDH2 in atherosclerosis pathogenesis. Bone marrow-derived macrophages were identified as the primary cell type responsible for the reduction in plaque load, as transplantation of bone marrow from $\mathrm{Aldh2^{-/- }} \mathrm{Ldlr}^{--}$mice into $\mathrm{Ldlr}^{-/}$mice was sufficient to prevent atherosclerotic plaque formation. As macrophage-mediated clearance of circulating lipids is a key contributor to ath-

Related Article: p. 252

Conflict of interest: The authors have declared that no conflict of interest exists.

Reference information: / Clin Invest. 2019;129(1):63-65. https://doi.org/10.1172/JCI125433.

erosclerotic progression (6), Zhong and colleagues next interrogated macrophage foam cell formation and the capacity of these cells to metabolize oxidized lipids (uptake, degradation, and efflux). Strik-

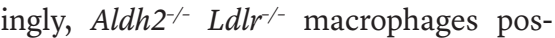
sessed an enhanced lysosomal cholesterol degradation capacity, which correlated with a reduction in foam cell formation. Importantly, Aldh2 deletion alone did not alter lysosomal degradation, indicating that the phenotype was LDLR-dependent. In a carefully designed set of experiments, the authors determined that autolysosome number was substantially increased in Aldh2 $2^{-/-} \mathrm{Ldlr}^{-/}$macrophages, suggesting an enhanced ability to clear lipid-laden endosomes. Notably, this protective effect was abolished when lysosomal $\mathrm{pH}$ was neutralized with bafilomycin A1, providing mechanistic links between ALDH2, lysosomal function, and foam cell formation. Collectively, these results suggested the contribution of a pathway that is dependent on ALDH2 and LDLR to the progression of atherosclerosis.

Next, Zhong et al. explored the underlying mechanism by which the interaction of ALDH2 and LDLR regulates foam cell formation and examined ALDH2 enzymatic activity, which was not different between WT and $\mathrm{Ldlr}^{-1}$ mice (5). This result suggested the potential for a direct interaction between the two proteins, and an interaction between LDLR and ALDH2 was experimentally confirmed by coimmunoprecipitation. The authors hypothesized that the interaction of LDLR and ALDH 2 prevented nuclear translocation, as observed in $\mathrm{Ldlr}^{--}$macrophages, and that this may be in part mediated by AMPK phosphorylation. This postulate was supported by previous work showing that AMPK regulates ALDH2 translocation to the nucleus, where it can act as a transcriptional repressor (7). Using transcriptomic RNA-Seq analysis in Aldh2/- $\mathrm{Ldlr}^{--}$and $L d r^{-/}$macrophages, the authors identified several genes that were regulated by 
A Normal macrophage clearance of lipids

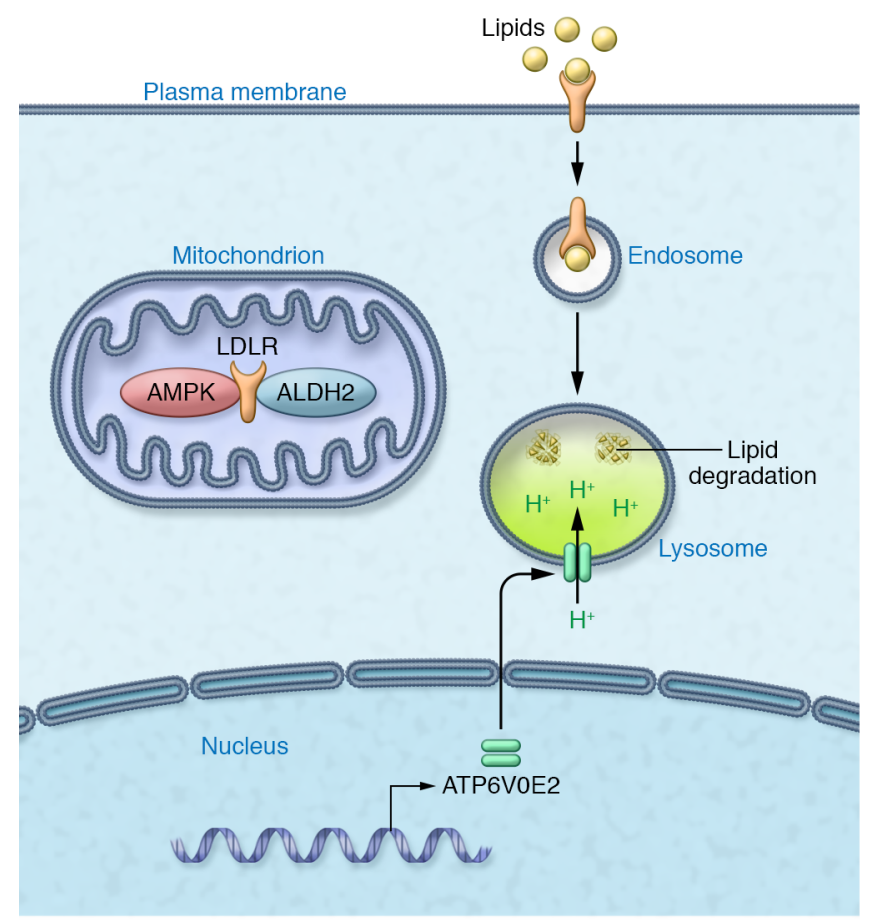

Efficient lysosomal lipid degradation
B $A L D H 2$ rs671 SNP-mediated foam cell formation

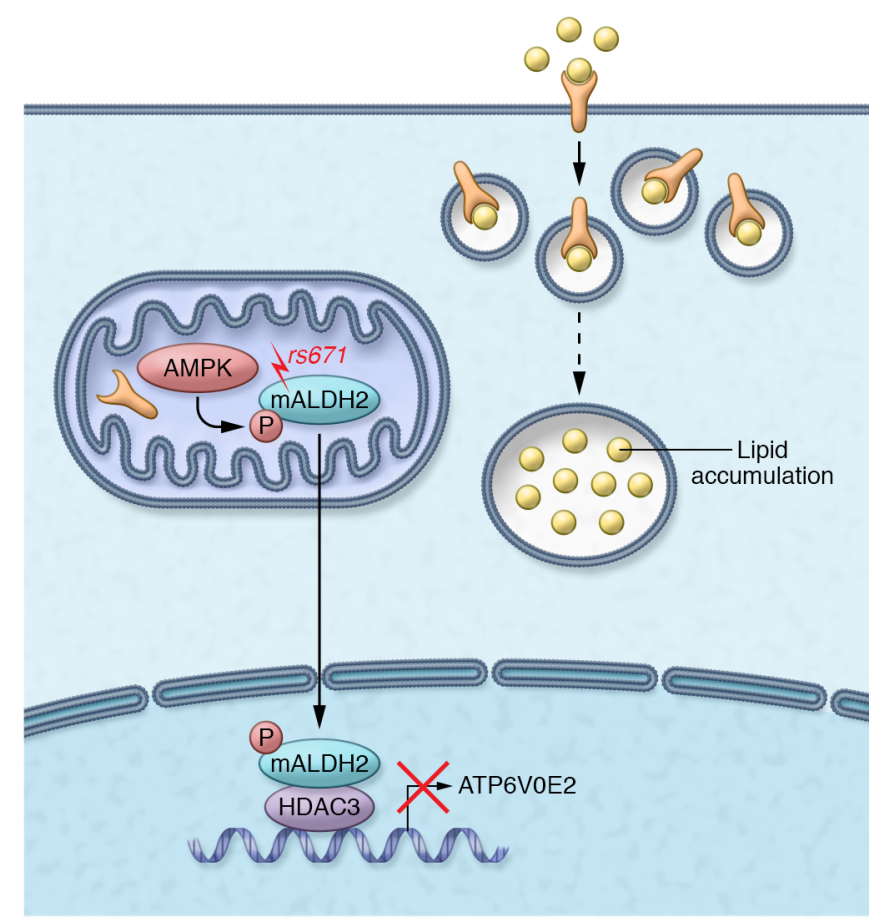

Lipid accumulation and foam cell formation
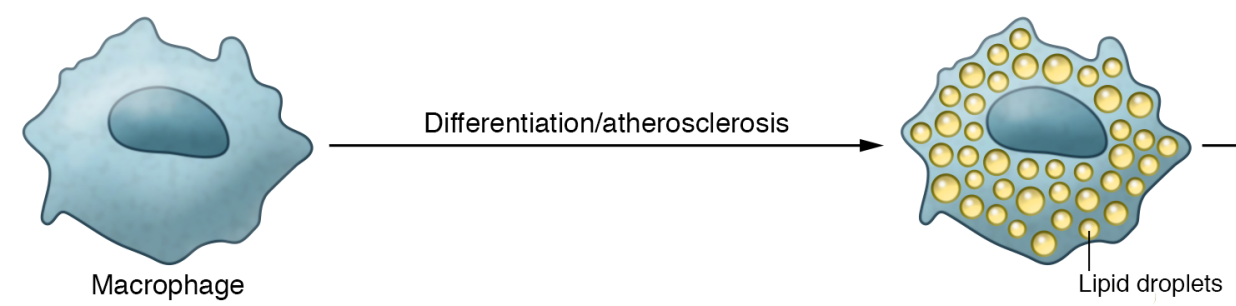

ROS

Necrotic factors

Proinflammatory cytokines

Figure 1. An ALDH2 SNP contributes to atherosclerosis. In this issue of the JCI, Zhong et al. (5) identify a pathway that reveals how the ALDH2 rs671 SNP promotes atherosclerosis. (A) The low-density lipoprotein receptor (LDLR) physically interacts with aldehyde dehydrogenase 2 (ALDH2) and 5' AMP-activated protein kinase (AMPK) to prevent AMPK-mediated phosphorylation and subsequent nuclear translocation of ALDH2. (B) The ALDH2 mutant protein (mALDH2 rs671 SNP) does not interact with LDLR, thereby removing the break on AMPK-mediated phosphorylation. Phospho-mALDH2 translocates to the nucleus and in an HDAC3-dependent fashion suppresses transcription of the lysosomal $\mathrm{H}^{+}$pump subunit, ATP6VOE2. ATP6VOE2 is critical for lysosomal acidification and autophagic clearance of lipids. The loss of this critical pathway accelerates foam cell formation and atherosclerosis.

ALDH2. Of particular interest, Atp6v0e2, a lysosomal proton pump that is required for proper lysosomal function and lipid clearance, was the third most upregulated gene. In a series of elegant molecular studies, Zhong et al. revealed that AMPK phosphorylation of ALDH2 promotes translocation to the nucleus, where its presence suppressed ATP6vOe2 transcription, all of which was prevented when LDLR was available to directly bind and sequester AMPK and ALDH2, preventing phosphorylation of ALDH2 and its nuclear translocation (Figure 1). This finding was important, as it provides a molecular mechanism that accounts for the authors' previous observations of lysosomal dysfunction mediating enhanced foam cell formation.

\section{Conclusions and clinical implications}

This serendipitous discovery was further extended toward clinical relevance, as expression of mutant ALDH2 rs671 mimicked the signaling observed with loss of LDLR. Moreover, the ALDH2 SNP prevented the direct interaction with LDLR and allowed for enhanced AMPK interaction and phosphorylation, resulting in ALDH2 rs671 nuclear translocation. This mechanism, in the context of earlier results, provides an explanation for the predisposition of this patient population to develop atherosclerosis and coronary heart disease. Convincingly, macrophages differentiated from peripheral blood monocytes isolated from human subjects carrying the $A L D H 2$ rs671 SNP displayed enhanced nuclear localization of ALDH2, diminished ATP6VOE2 lysosomal expression, and impaired cholesteryl ester hydrolysis. Furthermore, Zhong et al. showed that activation of AMPK by metformin treatment enhanced ALDH2 translocation and foam cell formation in human macrophages containing the $A L D H 2$ rs 671 SNP. This finding has significant clinical implications, as metformin is a regularly 
prescribed medication for individuals with diabetes, a comorbidity often associated with atherosclerosis. To this point, a recent meta-analysis indicated that the $A L D H 2$ rs671 SNP also correlated with the incidence of type 2 diabetes (8); therefore, prescription of metformin may further increase the risk of atherosclerosis in patients with the ALDH2 rs671 SNP and perhaps this novel signaling pathway is also linked to the pathogenesis of diabetes.

While the study by Zhong et al. provides causative evidence linking $A L D H 2$ to atherosclerosis (5), the exact role of $\mathrm{ALDH} 2$ in disease progression remains to be elucidated. There are several questions that have been brought to light by the current study and require further investigation to provide a more holistic view. First, what are the signaling events that lead to AMPK activation during atherosclerosis pathogenesis? As Zhong et al. mention, this appears to not be due to augmentation of conventional regulators (e.g., gene transcription, AMP/ATP levels). A better understanding of the upstream signaling events that lead to AMPK activation and ALDH2 phosphorylation could provide crucial mechanistic insight and novel therapeutic targets for $A L D H 2$ SNP carriers. Second, where does the ALDH2-LDLRAMPK interaction take place and what determines the subcellular localization of this interaction? Specifically, how do LDLR and AMPK enter the mitochondria to physically interact with ALDH2 and how does this modulate LDLR cycling that occurs with lipid uptake? These fundamental questions need to be addressed to place this novel signaling within the canonical LDLR lipid uptake pathway. Third, how is phosphorylated ALDH2 transported out of the mitochondria and into the nucleus and how is AMPKmediated phosphorylation regulated in the mitochondrial matrix environment? There are limited data to explain how AMPK may function in the mitochondria where the $\mathrm{pH}$ is significantly different than the cytosol, and what is the phosphatase counteracting AMPK phosphorylation. Fourth, whatother genes, beyond ATP6VOE2, does ALDH2 regulate and what is the impact on macrophage polarization, inflammation, and secretory factors. Further identification of the genes modulated by ALDH2 nuclear translocation and their impact on the vascular microenvironment could provide additional targets for translational application. Fifth, does a similar mechanism in the regulation of cholesterol metabolism exist outside of the macrophage? Hepatocyte clearance of LDL is the greatest contributor to circulating cholesterol levels. Does this pathway play a role in global lipid metabolism? Sixth, are patients with type 2 diabetes and the $A L D H 2$ SNP who are currently taking metformin at a greater risk of progressive atherosclerosis? Based on the results of Zhong et al., this appears to be an area in need of critical and immediate attention. Finally, what is the functional consequence of this novel ALDH2 signaling pathway in other disease states? Looking beyond atherosclerosis, it is interesting to note that $A L D H 2$ rs 671 is also associated with an increased risk of hypertension (9), diabetes mellitus (8), osteoporosis (10), and cancers $(11,12)$. Does nuclear translocation of ALDH2 contribute to the pathogenesis of these other disease states?

In summary, the current study by Zhong et al. (5) makes an important mechanistic link regarding the increased prevalence of atherosclerosis in individuals with the $A L D H 2$ rs671 SNP. While the complete underlying mechanisms remain to be elucidated, these findings provide the framework to further delineate the involvement of ALDH2 in not only atherosclerosis, but numerous other disease states of clinical significance.

\section{Acknowledgments}

This work was supported by NIH grants R01HL136954, R01HL123966, R01HL142271, P01HL134608 sub-5483, and P01DA037830 sub-8614 to JWE.
Address correspondence to: John W. Elrod, Center for Translational Medicine, Lewis Katz School of Medicine at Temple University, 3500 N. Broad Street, MERB 949, Philadelphia, Pennsylvania 19140, USA. Phone: 215.707.5480; Email: elrod@temple.edu.

1. Benjamin EJ, et al. Heart disease and stroke statistics-2018 update: a report from the American Heart Association. Circulation. 2018;137(12):e67-e492.

2. Defesche JC, Gidding SS, Harada-Shiba M, Hegele RA, Santos RD, Wierzbicki AS. Familial hypercholesterolaemia. Nat Rev Dis Primers. 2017;3:17093.

3. Ishibashi S, Brown MS, Goldstein JL, Gerard RD, Hammer RE, Herz J. Hypercholesterolemia in low density lipoprotein receptor knockout mice and its reversal by adenovirus-mediated gene delivery. J Clin Invest. 1993;92(2):883-893.

4. Han H, Wang H, Yin Z, Jiang H, Fang M, Han J. Association of genetic polymorphisms in $\mathrm{ADH}$ and $\mathrm{ALDH} 2$ with risk of coronary artery disease and myocardial infarction: a meta-analysis. Gene. 2013;526(2):134-141

5. Zhong S, et al. Acetaldehyde dehydrogenase 2 interactions with LDLR and AMPK regulate foam cell formation. J Clin Invest. 2019;129(1):252-267.

6. Moore KJ, Tabas I. Macrophages in the pathogenesis of atherosclerosis. Cell. 2011;145(3):341-355.

7. Choi JW, et al. Malondialdehyde inhibits an AMPK-mediated nuclear translocation and repression activity of ALDH2 in transcription. Biochem Biophys Res Commun. 2011;404(1):400-406.

8. Li GY, et al. Meta-analysis on the association of ALDH2 polymorphisms and type 2 diabetic mellitus, diabetic retinopathy. Int J Environ Res Public Health. 2017;14(2):E165.

9. Hasi T, Hao L, Yang L, Su XL. Acetaldehyde dehydrogenase 2 SNP rs671 and susceptibility to essential hypertension in Mongolians: a case control study. Genet Mol Res. 2011;10(1):537-543.

10. Hoshi $\mathrm{H}$, et al. Aldehyde-stress resulting from Aldh2 mutation promotes osteoporosis due to impaired osteoblastogenesis. JBone Miner Res. 2012;27(9):2015-2023.

11. Jiang Y, Zhang J, Wu Y, Wang J, Li L. Association between ALDH2 rs671 G>A polymorphism and gastric cancer susceptibility in Eastern Asia. Oncotarget. 2017;8(60):102401-102412.

12. Park JY, et al. Impact of smoking on lung cancer risk is stronger in those with the homozygous aldehyde dehydrogenase 2 null allele in a Japanese population. Carcinogenesis. 2010;31(4):660-665. 